\title{
Rice variety and processing: contribution to glycaemic response
}

\author{
U S Nisanka ${ }^{1}$, S Ekanayake ${ }^{1}$
}

(Index words: rice, parboiling, digestible carbohydrate, raw rice, glycaemic index)

\begin{abstract}
Introduction Glycaemic index (GI) of different varieties of rice (Oryza sativa L.) commonly consumed in Sri Lanka are not widely studied even though it is the staple food.
\end{abstract}

Objectives To determine the proximate compositions and the $\mathrm{Gl}$ of three different rice meals, namely $\mathrm{Bg} 360$ Samba (raw), Basmati At 405 (raw) and parboiled Nadu Bg 352.

Methods The study was a randomised cross over study using healthy volunteers $(n=11)$. GI was determined as the ratio between the incremental areas under curve obtained for the test food and the standard glucose (WHO/FAO method). Proximate compositions were determined by standard methods.

Results The $\mathrm{GI}$ value so obtained were, $66 \pm 8$ (medium GI) for Bg 360 Samba, $73 \pm 7$ (high GI) for Basmati At 405 and $40 \pm 4$ (low GI) for Nadu Bg 352. Parboiled variety had a significantly low $\mathrm{Gl}$ and the lowest peak glucose concentration. Significant differences were observed in insoluble dietary fibre (IDF), total dietary fibre (TDF) and moisture contents of the rice varieties. Non significant negative correlations between IDF $(r=-0.94, p=>0.05$, $\mathrm{n}=3)$ and TDF $(\mathrm{r}=-0.90, p=>0.05, \mathrm{n}=3)$ and $\mathrm{Gl}$ of the rice varieties were observed.

Conclusions Parboiled Nadu Bg 352 and Bg 360 Samba (raw) are of nutritional significance to individuals seeking to control the energy intake and glycaemic response.

Ceylon Medical Journal 2016; 61: 159-162

http://doi.org/10.4038/cmj.v61i4.8381

\section{Introduction}

The carbohydrate intake among Sri Lankan adults is over 14 portions of starch and $3 \cdot 5$ portions of added sugars daily. Fat consumption is reported to be modest (15-18\%) [1]. Over $70 \%$ of the population exceed the upper limit of the recommendations for starch intake with very little vegetable and fruit intake despite the variety and abundance of fruits and vegetables [1]. The study concluded that most nutrition-related diseases in the country may be closely related to unhealthy eating habits [1].

Glycaemic index (GI) is a method of classifying the blood glucose raising potential of carbohydrate rich foods [5]. Patients with metabolic disorders such as diabetes or hyperlipidaemia are recommended low-GI diets which give rise to a low and slow glycaemic response thus avoiding sudden fluctuations in the glycaemic response $[2,3]$. Despite being the staple food and the major contributor to carbohydrate intake, little data is available on the GI of local rice varieties [4-7]. Recently GI data on two imported basmati varieties available in the Sri Lankan market was reported [8].

In Sri Lanka rice (red or white) is consumed as raw or parboiled. Raw rice is obtained by milling paddy per se without subjecting to any pre-treatment whereas parboiled rice undergoes a hydrothermal treatment where rice is pre-cooked within the husk. During parboiling, starch gelatinises and during the subsequent drying stage undergoes partial retrogradation resulting in much harder paddy grains which can withstand the mechanical forces during milling. Consequently, the head rice percentage obtained by milling parboiled paddy is high. During the parboiling process water soluble vitamins tend to diffuse inward and even though thiamin undergoes partial degradation, parboiled milled rice has a higher vitamin content than raw milled rice [9]. The disintegration of rice grains upon cooking is considerably low and also the rice grains remain well-separated [10]. The quantity of solids leaching into the cooking water as well as the extent of solubilisation of the kernels upon cooking are significantly lowered due to resistance of the starch in the parboiled rice to swelling and solubilisation in hot water [10].

This study was aimed at determining the GI of three different varieties of rice, Bg 360 Samba-raw white, At

${ }^{1}$ Department of Biochemistry, Faculty of Medical Sciences, University of Sri Jayewardenepura, Sri Lanka.

Correspondence: SE, e-mail: <sagarikae@hotmail.com>. Received 11 April 2016 and revised version accepted 9 July 2016. and reproduction in any medium, provided the original author and source are credited. 
405 Basmati-raw white [Samurdhi] and Bg $352 \mathrm{Nadu}$ - parboiled white which are commonly available and consumed in Sri Lanka.

\section{Methods}

The rice varieties Bg 352 Nadu and Bg 360 Samba bulk paddy samples were obtained from CIC Agribusiness (Pvt) Ltd, Sri Lanka. Basmati At 405 (Lanka Samurdhi raw white) was obtained from the Rice Research Station, Ambalantota, Sri Lanka. Nadu paddy was parboiled by steeping paddy in water at room temperature and heating until the husk of paddy grains started to split (cold soak method). The paddy was sun dried thoroughly prior to milling. All bulk samples of paddy were milled (Rice Huller LM 24-2C, China), polished twice (N 70 HP type, China) and destoned.

Rice was washed and mixed with water in the ratios mentioned in Table 1 and cooked in a rice cooker (Kapok, China). Cooked rice was dried in an oven $\left(50^{\circ} \mathrm{C}\right)$, ground to a fine powder (IKA ${ }^{\circledR} \mathrm{A} 11$ basic, Brazil), sieved $(0.315 \mathrm{~mm})$ and stored at $4{ }^{\circ} \mathrm{C}$ until analysis was made. The moisture content was measured by AOAC official method [11]. The digestible carbohydrate content was measured by Holm's method [12] and insoluble (IDF) and soluble dietary fibres (SDF) were determined by the method of Asp et al., [13].

\section{Table 1. Cooking details of the rice varieties}

\begin{tabular}{lcc}
\hline Rice variety & $\begin{array}{c}\text { Water ml /cup } \\
(240 \mathrm{ml}) \text { of rice }\end{array}$ & $\begin{array}{c}\text { Cooking } \\
\text { time / min }\end{array}$ \\
\hline $\begin{array}{l}\text { Bg 360 Samba } \\
\text { (raw white) }\end{array}$ & 360 & 30 \\
$\begin{array}{l}\text { Bg 352 Nadu } \\
\text { (parboiled white) }\end{array}$ & 480 & 45 \\
$\begin{array}{l}\text { At 405 Basmati } \\
\text { (raw white) }\end{array}$ & 240 & 20 \\
& & \\
\end{tabular}

The study was a randomised cross over study. Eleven healthy volunteers ( 6 males and 5 females) not on any medical treatment $(\mathrm{BMI}=18-25$, age 20-30, fasting blood glucose level $\square 110 \mathrm{mg} / \mathrm{dl}$, peak blood glucose level after consuming $50 \mathrm{~g}$ of glucose $\square 180 \mathrm{mg} / \mathrm{dl}$ ) were selected. GI values were estimated according to the slightly modified WHO/FAO (1998) and Wolever et al, 2006 methods $[14,15]$. Each volunteer was served the standard twice and each test food once in random order on separate days. Glucoline (GSK Glaxco Wellcome Ceylon Ltd. Sri Lanka) dextrose monohydrate was used as the standard. The volunteers were served with rice portions corresponding to $50 \mathrm{~g}$ digestible carbohydrate ( $\mathrm{Bg} 360 \mathrm{Samba}(225 \mathrm{~g}), \mathrm{Bg}$ $352 \mathrm{Nadu}(200 \mathrm{~g})$ and At 405 Basmati $(150 \mathrm{~g})$ ) with $25 \mathrm{~g}$ of coconut sambol (scraped fresh coconut kernel mixed with chilly, onion, salt and lime) and $250 \mathrm{ml}$ of water. Finger prick, capillary blood samples were collected at fasting and 30 - 120 min after ingestion of test/ standard food (Onetouch Ultrasoft lancets Lifescan Inc., USA). Blood glucose was estimated with the glucose oxidase enzymatic kit (GOD-PAP, Biolabo, France). Incremental area under the blood glucose curve (IAUC) was determined for each individual for rice and glucose. GI was calculated as a ratio between the IAUC of the test food and the standard. Glycaemic Load (GL) was estimated by multiplying the GI by the number of net carbohydrates in a given serving.

All procedures involving human participants were approved by the Ethics Committee of Faculty of Medical Sciences, University of Sri Jayewardenepura, Sri Lanka (Approval number 504/10). Written informed consent was obtained from all participants.

Students' t-test was used to determine the significant differences between the digestible carbohydrate, dietary fibre contents and GI of the three rice varieties. Regression analysis was used to find correlations between GI and amount of dietary fibre present in the $50 \mathrm{~g}$ available carbohydrate portion (Minitab version 15 and Microsoft Excel 2007).

\section{Results}

Moisture, digestible carbohydrate, dietary fibre and GI data are given in Table 2. Moisture content of the cooked rice varieties ranged from $57.7-72.1 \%$ and were significantly different $(p<0.05)$ with $\mathrm{Nadu} \mathrm{Bg} 352$ (parboiled) and Samba Bg 360 having more (69.7-72\%) moisture. Parboiled Nadu had the highest digestible carbohydrate content $(81.0 \%$, dry basis) while Samba Bg 360 had the lowest (77.9\%, dry basis). There was no significant difference in carbohydrate contents. Parboiled Bg 352 Nadu rice had significantly high $(p<0.05)$ amount of IDF (4.3\%) and total dietary fibre (TDF) (7.1\%). Basmati At 405 contained the lowest dietary fibre.

Nadu Bg 352 had a significantly low GI compared to the other two rice varieties and was classified as low GI (40) category. In addition, $\mathrm{Nadu}$ (Bg 352) elicited a low and consistent glycaemic response among the participants. Notably, all individuals showed either low (82\%) or medium (18\%) GI values for this particular Nadu (Bg 352) variety. $\mathrm{Bg} 360$ Samba elicited a medium GI (66) and At 405 Basmati, a locally developed variety elicited a high GI (73). Regression analysis showed a non significant negative correlation between IDF $(\mathrm{r}=-0.94, p=>0.05$, $\mathrm{n}=3), \mathrm{n}$ TDF $(\mathrm{r}=-0.90, p=>0.05, \mathrm{n}=3)$ and GI and a non significant positive correlation between $\operatorname{SDF}(\mathrm{r}=0.38$, $p=>0.05, \mathrm{n}=3$ ) and GI of the rice varieties. 
Table 2. Moisture, digestible carbohydrate, dietary fibre contents and

GI of rice varieties ( $g / 100 \mathrm{~g} \mathrm{DW})$

\begin{tabular}{|c|c|c|c|c|c|c|}
\hline \multirow[t]{2}{*}{ Parameter } & \multicolumn{2}{|c|}{ At 405 Basmati } & \multicolumn{2}{|c|}{ Bg 352 Nadu } & \multirow{2}{*}{$\begin{array}{l}\text { Bg } 360 \\
\text { Mean }\end{array}$} & \multirow{2}{*}{$\begin{array}{l}\text { Samba } \\
\text { SD }\end{array}$} \\
\hline & Mean & $S D$ & Mean & $S D$ & & \\
\hline $\begin{array}{l}\text { Moisture of freshly cooked } \\
\text { sample (WB)* }\end{array}$ & 57.7 & $1^{\mathrm{a}}$ & 69.7 & $0.4^{\mathrm{b}}$ & 72.1 & $0.7^{\mathrm{c}}$ \\
\hline Digestible carbohydrate* & 78.6 & $5^{\mathrm{a}}$ & 81.0 & $2^{\mathrm{a}}$ & 77.9 & $3^{\mathrm{a}}$ \\
\hline Insoluble dietary fibre* & 1.7 & $0.2^{\mathrm{a}}$ & 4.3 & $1^{\mathrm{b}}$ & 3.0 & $0.4^{\mathrm{c}}$ \\
\hline Soluble dietary fibre** & 2.7 & $0.7^{\mathrm{a}}$ & 2.8 & $0.4^{\mathrm{a}}$ & 2.9 & $0.2^{\mathrm{a}}$ \\
\hline Total fibre & $4.4^{\mathrm{a}}$ & & $7.1^{\mathrm{b}}$ & & $5.9^{c}$ & \\
\hline Glycaemic index $( \pm \text { SEM })^{\#}$ & 73 & $23^{\mathrm{a}}(\mathrm{H})$ & 40 & $13^{\mathrm{b}}(\mathrm{L})$ & 66 & $27^{\mathrm{a}}(\mathrm{M})$ \\
\hline Glycaemic load & 57 & & 32 & & 51 & \\
\hline
\end{tabular}

$\mathrm{SD}$ - standard deviation; SEM - standard error of mean; WB - wet basis; DW - dry weight; $*_{n}=6$; * $\mathrm{n}=4$; \#n=11; different superscripts in a row indicate significant difference at $95 \%$ confidence interval. $\mathrm{H}, \mathrm{M}$ and $\mathrm{L}-$ high, medium and low GI respectively.

The portion sizes of $\mathrm{Bg} 360$ Samba and $\mathrm{Nadu} \mathrm{Bg} 352$ rice meals were considered adequate by all the participants whereas the At 405 Basmati portion size was considered insufficient by $64 \%$. All three rice varieties elicited high GL values i.e. GL 20 (Table 2). Basmati At 405 exerted high GL in all (100\%) individuals whereas for Samba (Bg 360 ) showed intermediate GL in $18 \%$ of individual and high GL $82 \%$. In the case of Nadu (Bg 352), low GL was shown in $9 \%$ intermediate GL $36 \%$ and high GL in 55\%.

When considering the glycaemic response of the rice meals following consumption, Basmati (At 405) which had the highest GI value shows the highest peak and $\mathrm{Samba} \mathrm{Bg} 360$ which had a medium GI value shows an inter-mediate peak glucose concentration, with low GI Nadu eliciting the lowest mean peak glucose concentration (Figure 1).

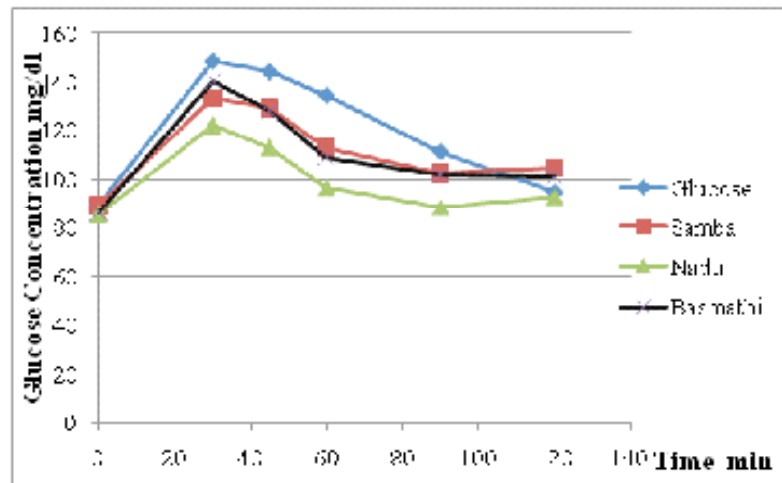

Figure 1. Blood glucose response curves of the three rice meals and glucose (control).

\section{Discussion}

Among the rice varieties studied, parboiled $\mathrm{Bg}$ 352 Nadu rice had the highest IDF and TDF indicating

Vol. 61, No. 4, December 2016 increased amount of undigestible carbohydrates which may include the retrograded starch due to the parboiling process. Though a significant difference in the distribution of IDF contents of the three rice varieties was observed, no significant difference $(p>0.05)$ was observed in SDF which also signifies the contribution to IDF from parboiling process. Research on red rice meals concluded that the glycaemic response of the meals showed a significant negative correlation with the dietary fibre content. However, the dietary fibre in the red rice meal was from multiple sources of accompaniments in the meal [4].

Parboiled Nadu elicited a low GI and the data agrees with literature where parboiled rice has elicited lower GI compared to the unparboiled (raw) counterparts $[5,6]$. The GI of the raw (unparboiled) Bg $352 \mathrm{Nadu}$ is reported to be $67 \pm 3$ [9]. The low GI of parboiled Nadu (Bg 352) thus can be attributed to comparatively high fibre content with retrograded starch which would have resulted slow gastric emptying, decreased digestion and absorption leading to low postprandial glucose levels. Therefore, this particular Nadu Bg 352 variety is nutritionally suitable for those with diabetics mellitus. Bg 360 Samba (medium GI) can also be recommended for patients with diabetes mellitus if accompanied by vegetables/legumes. In contrast to low or medium GI values reported for Basmati rice, At 405 Basmati, had a high GI [16]. However, a low GI was elicited by commercially available Indian and Pakistani basmati rice according to a recent study [8]. Notably the GI values reported for these basmati rice are higher than that of parboiled Nadu $(\mathrm{GI}=40)$ as found in the present study. The differences in starch structure in different rice varieties may contribute to the differences in GI. Hettiaratchi et al., had shown that accompanying foods could reduce the the GI of a rice meal [4]. In this study rice was served with coconut sambol $(25 \mathrm{~g})$. The effect caused by $25 \mathrm{~g}$ of coconut sambol was common for all three meals and thus would not have affected the GI of the three meals. 
The differences between the GI values obtained for rice in published research could be mainly due to the differences in the food itself, processing and the methodologies employed in calculating the carbohydrate content. In most GI research, the available carbohydrate content is determined by the difference leading to overestimation of available carbohydrate content [17]. Moreover, resistant starches are not included in the difference method. Thus when they are present will be mistakenly counted as glycaemic or available carbohydrate [18]. In contrast, the available carbohydrate content was directly measured in this study.

Feedback by the volunteers regarding the adequacy of portion size and palatability of the meal was highly subjective. The portions of $\mathrm{Bg} 360 \mathrm{Samba}$ and Nadu Bg 352 rice meals were considered adequate. At 405 Basmati portion size was insufficient for $64 \%$. At 405 Basmati had the lowest moisture and dietary fibre content among the three rice varieties. The moisture content of a food thus is a major factor that determines the edible portion size and low dietary fibre lowers the satiety level. Majority of the individuals (91\%) in the cohort preferred Basmati (At 405) highlighting the general preference for more refined foods. Nadu Bg 352 was disliked by $64 \%$ of the individuals.

Glycaemic load (GL) takes into account the amount of available carbohydrates present in the meal portion. In all individuals the highest GL was for Basmati At 405 . This is indicative of highly available carbohydrate. Since GL is a product of two variables, GL can be reduced either by reducing GI or reducing available carbohydrate content. In this respect consumption of a large quantity of available carbohydrate (portion size) of a low GI food can result in similar GL to consumption of considerably low available carbohydrate (portion size) of a high GI food. According to Wolever, the health effects of reducing diet GL cannot be predicted, and more importantly these health effects should not be equated with the effects of reducing diet GI [15]. Of the three rice varieties, parboiled rice which had a low GI and a comparatively lower GL can be recommended in the dietary regime of patients with diabetes mellitus. Consumption of parboiled rice is a practice that should be promoted among people.

In conclusion, due to the low peak glucose concentration, slower rise in postprandial blood glucose level, low GI and the low carbohydrate load compared to other rice varieties, consumption of parboiled Nadu Bg 352 as a low GI food can be recommended.

\section{Funding}

This work was supported by IPICS, Uppsala University, Sweden (grant no SRI:07) and the National Research Council of Sri Lanka (grant no NRC-05-03).

\section{Conflicts of interest}

There are no conflicts of interest.

\section{References}

1. Jayawardena R, Byrne NM, Soares MJ, et al. Food consumption of Sri Lankan adults: an appraisal of serving characteristics. Public Health Nutr 2013; 16: 653-8. DOI: http://dx.doi.org/10.1017/S1368980012003011

2. Wolever TMS, Jenkins DJA, Jenkins AL, et al. The glycemic index: methodology and clinical implications. Am J Clin Nutr 1991; 54: 846-54.

3. Wolever TMS, Katzman-Rellea L, Jenkins AL, et al. Glycaemic index of 102 complex carbohydrate foods in patients with diabetes. Nutr Res 1994; 14: 651-69.

4. Hettiaratchi UPK, Ekanayake S, Welihinda J. Do Sri Lankan meals help decrease blood glucose response? Ceylon Med J 2009; 54: 39-43.

5. Hettiarachchi P, Jiffry MTM, Jansz ER, et al. Glycaemic indices of different varieties of rice grown in Sri Lanka. Ceylon Med J 2008; 46: 11-4.

6. Pathiraje PMHD, Madhujith WMT, Chandrasekara A, et al. The effect of rice variety and parboiling on in vivo glycaemic response. Tropical Agricultural Research 2010; 22: 26-33.

7. Chan HMS, Brand-Miller JC, Holt SHA, et al. The glycaemic index values of Vietnamese foods. Eur J Clin Nutr 2001; 55: 1076-83.

8. Gunathilaka MDTL and Ekanayake S. Effect of different cooking methods on glycaemic index (GI) of Indian and Pakistani basmati rice varieties. Ceylon Med J 2015; 60: 57-61.

9. Padua $\mathrm{AB}$, Juliano BO. Effect of parboiling on thiamin, protein and fat of rice. J Sci Food Agric 1974; 25: 697-701.

10. Priestley RJ. Studies on parboiled rice: Part 1-Comparison of the characteristics of raw and parboiled rice. Food Chem 1976; 1: 5-14.

11. Association of Official Analytical Chemist (14.06). Official methods of analysis of the AOAC, 14th ed. Washington, DC: AOAC, 1984.

12. Holm J, Drews A, Asp NG. A rapid method for the analysis of starch. Starch / Starke 1986; 38: 224-6.

13. Asp NG, Hallmer H, Siljestrom M. Rapid enzymatic assay of insoluble and soluble dietary fibre. J Agric Food Chem 1983; 31: 476-8.

14. FAO/WHO. Carbohydrates in human nutrition: report of a joint FAO/WHO expert consultation. 1998. Food and Agricultural Organization of the United Nations, Rome, Italy.

15. Wolever TMS. The Glycaemic Index - A physiological classification of dietary carbohydrate. London: Biddles Ltd, King's Lynn, 2006.

16. Aston LM, Gambell JM, Lee DM, et al. Determination of the glycaemic index of various staple carbohydrate-rich foods in the UK diet. Eur J Clin Nutr 2008; 62: 279-85.

17. Thadhani VM, Jansz ER, Peiris H. The digestible carbohydrate and dietary fibre contents of common Sri Lankan plant foodstuffs. J Nat Sci Foundation Sri Lanka 2000; 28: 253-64.

18. Food and Agriculture Organization of the United Nations / World Health Organization. 1997. (http://www.fao.org/ docrep/W8079E/w8079e0a.htm\#definition of glycemic index (gi)) (accessed March 2013) 